\title{
Letrozole Therapy Alone or in Sequence with Tamoxifen in Women with Breast Cancer
}

\author{
The BIG 1-98 Collaborative Group*
}

ABSTRACT

\begin{abstract}
The members of the writing committee (Henning Mouridsen, M.D., chair, Anita Giobbie-Hurder, M.S., Aron Goldhirsch, M.D., Beat Thürlimann, M.D., Robert Paridaens, M.D., Ian Smith, M.D., Louis Mauriac, M.D., John F. Forbes, F.R.A.C.S., M.S., Karen N. Price, B.S., Meredith M. Regan, Sc.D., Richard D. Gelber, Ph.D., and Alan S. Coates, M.D.) assume full responsibility for the overall content and integrity of the article. The affiliations of the members of the writing committee are listed in the Appendix. Address reprint requests to the International Breast Cancer Study Group (IBCSG) Coordinating Center, Effingerstr. 40, 3008 Bern, Switzerland, or at ibcsg18_BIGl-98@ fstrf.org.
\end{abstract}

*Members of the Breast International Group (BIG) 1-98 Collaborative Group are listed in Section 1 in the Supplementary Appendix, available with the full text of this article at NEJM.org.

N Engl J Med 2009;361:766-76.

Copyright @ 2009 Massachusetts Medical Society.

\section{BACKGROUND}

The aromatase inhibitor letrozole, as compared with tamoxifen, improves diseasefree survival among postmenopausal women with receptor-positive early breast cancer. It is unknown whether sequential treatment with tamoxifen and letrozole is superior to letrozole therapy alone.

\section{METHODS}

In this randomized, phase 3, double-blind trial of the treatment of hormone-receptor-positive breast cancer in postmenopausal women, we randomly assigned women to receive 5 years of tamoxifen monotherapy, 5 years of letrozole monotherapy, or 2 years of treatment with one agent followed by 3 years of treatment with the other. We compared the sequential treatments with letrozole monotherapy among 6182 women and also report a protocol-specified updated analysis of letrozole versus tamoxifen monotherapy in 4922 women.

\section{RESULTS}

At a median follow-up of 71 months after randomization, disease-free survival was not significantly improved with either sequential treatment as compared with letrozole alone (hazard ratio for tamoxifen followed by letrozole, 1.05; 99\% confidence interval [CI], 0.84 to 1.32; hazard ratio for letrozole followed by tamoxifen, 0.96; $99 \% \mathrm{CI}, 0.76$ to 1.21 ). There were more early relapses among women who were assigned to tamoxifen followed by letrozole than among those who were assigned to letrozole alone. The updated analysis of monotherapy showed that there was a nonsignificant difference in overall survival between women assigned to treatment with letrozole and those assigned to treatment with tamoxifen (hazard ratio for letrozole, $0.87 ; 95 \% \mathrm{CI}, 0.75$ to $1.02 ; \mathrm{P}=0.08$ ). The rate of adverse events was as expected on the basis of previous reports of letrozole and tamoxifen therapy.

\section{CONCLUSIONS}

Among postmenopausal women with endocrine-responsive breast cancer, sequential treatment with letrozole and tamoxifen, as compared with letrozole monotherapy, did not improve disease-free survival. The difference in overall survival with letrozole monotherapy and tamoxifen monotherapy was not statistically significant. (ClinicalTrials.gov number, NCT00004205.) 
F OR DECADES, THE STANDARD ADJUVANT endocrine therapy for postmenopausal women with hormone-receptor-positive early breast cancer was tamoxifen, taken for 5 years, a treatment that improved disease-free survival and reduced the number of deaths from breast cancer. ${ }^{1}$ More recently, reports from the Breast International Group (BIG) 1-98 trial2,3 and the Arimidex, Tamoxifen, Alone or in Combination trial (ATAC; ClinicalTrials.gov number, NCT00849030) $)^{4,5}$ showed that 5 years of adjuvant therapy with an aromatase inhibitor alone improved disease-free survival as compared with 5 years of tamoxifen therapy; other large studies showed that switching to an aromatase inhibitor after initial treatment with tamoxifen improved survival. 6-12 A meta-analysis ${ }^{13}$ of trials of initial and sequential strategies supported the recommendation in guidelines that an aromatase inhibitor should be included in adjuvant therapy for postmenopausal women with endocrine-responsive early breast cancer. $^{14-16}$

In the BIG 1-98 study, we compared monotherapy with tamoxifen, monotherapy with an aromatase inhibitor, and two sequential treatments: tamoxifen followed by an aromatase inhibitor (for which models predicting contradictory outcomes have been published ${ }^{17,18}$ ) and an aromatase inhibitor followed by tamoxifen. Initial results from the BIG 1-98 trial showed that the aromatase inhibitor letrozole given alone, as compared with tamoxifen given alone, reduced the risk of recurrent disease, especially at distant sites. ${ }^{2}$ In this report, we present the results of the comparison of each sequential treatment with letrozole monotherapy. We also present a protocol-defined updated analysis of the comparison between 5 years of monotherapy with tamoxifen and 5 years of monotherapy with letrozole.

METHODS

\section{STUDY DESIGN}

The trial design has been described previously. 2,3,19 Briefly, the BIG 1-98 trial is a randomized, phase 3, double-blind trial involving postmenopausal women with estrogen-receptor-positive or progesterone-receptor-positive early breast cancer. Initially, from March 1998 through March 2000, women were randomly assigned to receive only letrozole (Femara, Novartis), $2.5 \mathrm{mg}$ daily, or only tamoxifen, $20 \mathrm{mg}$ daily, for 5 years; however, from April 1999 through May 2003, women were randomly assigned to one of four study treatments: only tamoxifen for 5 years, only letrozole for 5 years, letrozole for 2 years followed by tamoxifen for 3 years, or tamoxifen for 2 years followed by letrozole for 3 years (Fig. 1).

The primary end point was disease-free survival, defined as the time from randomization to the first of any of the following events (hereinafter called primary-end-point events): recurrence of the disease at a local, regional, or distant site; a new invasive cancer in the contralateral breast; any second (nonbreast) cancer; or death without a previous cancer event. Other end points included time to the recurrence of breast cancer (including invasive contralateral breast cancer but not considering second [nonbreast] cancers and with censoring of deaths that were not associated with a previous cancer event); time to distant recurrence, defined as the time from randomization to the recurrence of breast cancer at a distant site; and overall survival.

The 2005 results, ${ }^{2}$ which showed the superiority of letrozole over tamoxifen, led to the recommendation by the data and safety monitoring committee of the International Breast Cancer Study Group (IBCSG), and a decision by the BIG 1-98 steering committee, to inform women in the tamoxifen-monotherapy group of their treatment, thereby allowing informed decisions to be made about their future care. An amendment of the protocol in April 2005 allowed for the provision of letrozole to any patient assigned to tamoxifen monotherapy who was free of disease, was receiving tamoxifen, and wished to cross over to letrozole (selective crossover). With respect to the three groups whose treatment regimens included letrozole, the double-blind nature of the study remained in effect.

\section{STUDY PROCEDURES}

Clinical assessments were performed at baseline, every 6 months for the first 5 years, and yearly thereafter. Six-month supplies of study drugs that were identical in appearance and packaging were dispensed at each semiannual study visit for 5 years. Hematologic and blood chemical measurements and bilateral mammographic studies were per- 


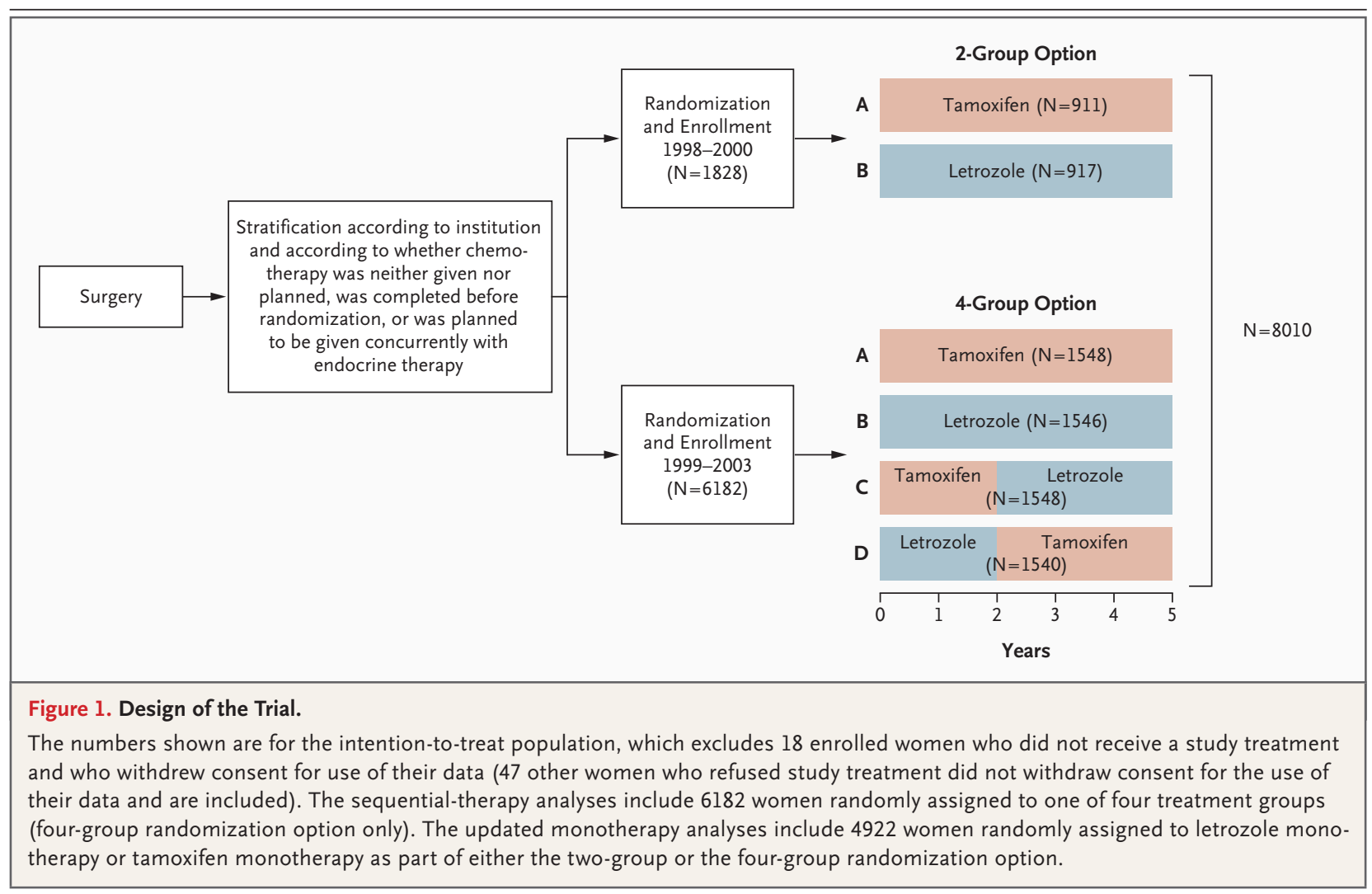

formed at baseline and were repeated as medically indicated. Adverse events, including a cerebrovascular accident or transient ischemic attack, cardiac ischemic infarction, angina requiring percutaneous transluminal coronary angioplasty, angina requiring coronary-artery bypass grafting, any thromboembolic event, other cardiovascular events, hypercholesterolemia (usually assessed when the patient was not fasting), bone fracture, vaginal bleeding, nausea, vomiting, hot flashes, and night sweats, were listed on the case-report forms and graded according to the Common Toxicity Criteria (version 2) of the National Cancer Institute at each study visit. Other adverse events were recorded in free-text format on the case-report forms. Serious adverse events were reported promptly, in accordance with regulatory requirements.

The IBCSG was responsible for the design and coordination of the study, the collection and management of the data, the medical review, the analysis of the data, and the reporting of the results. The members of the trial steering committee (see Section 1 in the Supplementary Appendix, available with the full text of this article at NEJM. org) reviewed the manuscript and were respon- sible for the decision to submit it for publication. The ethics committee and relevant health authorities at each participating institution approved the study protocol. All women gave written informed consent. The data and safety monitoring committee received safety data semiannually throughout the trial and reviewed three predefined interim efficacy analyses and the final efficacy analysis. Novartis, the manufacturer of letrozole, distributed the study drugs and provided financial support but imposed no restrictions on the investigators with respect to trial data. The IBCSG Statistical Center had full access to the trial database (which included all data related to the trial, except for study-treatment assignment) and to the study-treatment-assignment database, but the IBCSG Data Management Center had access to the trial database only. The manuscript was prepared by the members of the writing committee, who made the final decisions about the content. Members of the steering committee (including a minority representation from Novartis) reviewed the article and suggested changes. The chair of the writing committee vouches for the accuracy and completeness of the data. The data 
were analyzed by statisticians at the IBCSG Statistical Center.

The final efficacy analysis of the sequential treatments was reviewed by the data and safety monitoring committee in October 2008 and released to the steering committee when a protocoldefined number of primary-end-point events had occurred. At the same time, the planned 10-year efficacy update of the monotherapy treatments was performed.

\section{STATISTICAL ANALYSIS}

The evaluation of sequential treatments involved only the women who participated in the four-group randomization option. To ensure adequate power, statistical considerations were based on primaryend-point events that occurred after the fifth 6-month supply of study medication was dispensed - that is, after the agents were changed in the sequential treatments (approximately 2 years after randomization). The objective was to assess the superiority of switching endocrine agents as compared with continuing the initial agent. For each of the two pairwise comparisons (tamoxifen followed by letrozole vs. tamoxifen and letrozole followed by tamoxifen vs. letrozole), we calculated that at least 331 primary-end-point events after the switch in treatment would be needed for the study to have $80 \%$ power to detect a $29 \%$ reduction in the risk of a primary-end-point event after the switch. (Section 2 in the Supplementary Appendix shows the results of the treatment comparisons evaluated after the time of the switch in treatment.)

In 2005, on the basis of emerging data from the BIG 1-98 trial and other trials, the data and safety monitoring committee recommended that the steering committee revise the statistical-analysis plan to include five additional pairwise treatment comparisons, with analyses starting from the time of randomization. An amendment activated in April 2005, before any evaluation of results for the sequential-treatment groups was performed, specifies the comparisons, which include the two that are most clinically relevant: comparisons of each sequential treatment with 5 years of letrozole monotherapy. These comparisons are the main focus of this report. Post hoc power calculations showed that if the true reduction in the risk of a primary-end-point event was at least $26.7 \%$, there was an $80 \%$ chance that the $99 \%$ confidence interval would exclude a hazard ratio of 1.00. (Section 3 in the Supplementary Appendix shows the results of the other three pairwise treatment comparisons specified in the amendment of April 2005.)

Analyses were performed according to the intention-to-treat principle. Kaplan-Meier ${ }^{20}$ estimates of the time-to-event end points were calculated. A Cox proportional-hazards regression analysis $^{21}$ (stratified according to chemotherapy use, on the basis of the randomization stratum [Fig. 1]) was used to estimate P values and hazard ratios, with $99 \%$ confidence intervals to account for the five comparisons described in the amendment of April 2005. We used cumulativeincidence estimates ${ }^{22}$ to control for competing risks. The significance of differences in the incidence of adverse events among the four treatment groups was assessed with the use of Fisher's exact test; these analyses were not adjusted for multiple comparisons.

The study protocol specified that an updated analysis of the comparison of letrozole monotherapy with tamoxifen monotherapy be performed 10 years after the beginning of the trial. We therefore updated the previous analysis ${ }^{3}$ of the 4922 women who were assigned to one of the two monotherapy groups either as part of the twogroup or as part of the four-group randomization option. For this analysis, the Cox models were stratified both according to chemotherapy use and according to randomization option, and $95 \%$ confidence intervals were calculated. Among the 2459 women assigned to tamoxifen monotherapy, 619 (25.2\%) selectively crossed over to letrozole before a primary-end-point event occurred, and follow-up after the crossover accounted for $7.2 \%$ of total patient-years of follow-up. Women who selectively crossed over were more likely to have node-positive disease than those who continued to receive tamoxifen (46.9\% vs. $29.0 \%$ ). Crossovers occurred between 3 and 5 years after the start of therapy, and the average duration of letrozole therapy after crossover was 18 months. In addition to intentionto-treat analyses, exploratory analyses were performed in which data were censored at the time of crossover.

\section{RESULTS}

\section{ANALYSIS OF SEQUENTIAL TREATMENT}

\section{Clinical Characteristics}

A total of 8028 women were enrolled in the BIG 1-98 trial; 18 withdrew consent and did not receive treatment, leaving an intention-to-treat pop- 
ulation of 8010 (Fig. 1). The sequential-treatment analyses were performed on the basis of the 6182 women in the intention-to-treat population who were randomly assigned to a treatment group as part of the four-group option. This cohort included 3604 women $(58.3 \%)$ with node-negative disease, 3480 (56.3\%) in whom the primary tumor was less than $2 \mathrm{~cm}, 3782$ (61.2\%) who underwent breast-conserving surgery, and 4596 (74.3\%) who received no adjuvant or neoadjuvant chemotherapy. The median age at randomization was 61 years (range, 38 to 89). Clinical characteristics were well balanced across the four treatment groups (data not shown). The median follow-up period for the sequential-treatment analyses was 71 months. The database for this report was locked on July 2, 2008.

\section{Efficacy}

Figure 2 shows the hazard ratios, with $99 \%$ confidence intervals, for the comparisons of each of the sequential treatments with letrozole monotherapy with respect to the study end points. Differences between the treatment groups were not significant. The Kaplan-Meier estimates of the percentage of patients who remained diseasefree at 5 years after randomization were $87.9 \%$ in the group that was assigned to letrozole alone, $87.6 \%$ in the group that was assigned to letrozole followed by tamoxifen, and $86.2 \%$ in the group that was assigned to tamoxifen followed by letrozole. The estimated 5-year rate of disease-free survival for women in the tamoxifen-monotherapy group was $84.6 \%$ on the basis of the intention-totreat analysis in which 612 of the 1548 women in the tamoxifen-monotherapy group (39.5\%) crossed over to letrozole. Section 3 of the Supplementary Appendix shows the Kaplan-Meier curves for disease-free survival in all four groups, the sites of first primary-end-point events, and the hazard ratios for the five pairwise comparisons.

Figure 3 shows the cumulative incidence of the recurrence of breast cancer among women in each of the two sequential-regimen groups as compared with the letrozole-monotherapy group, with second, nonbreast primary cancers and deaths without a recurrence of breast cancer considered as competing events. The risk of a recurrence of breast cancer with tamoxifen followed by letrozole did not differ significantly from the risk with letrozole alone (Fig. 3A and 3C). There was no dif- ference in the outcome between women who were assigned to letrozole alone and those who were assigned to letrozole followed by tamoxifen, regardless of nodal status (Fig. 3B and 3D).

\section{Safety}

Section 4 in the Supplementary Appendix shows the adverse events that occurred among women who were randomly assigned to a treatment group as part of the four-group option, according to time (years 1 and 2, 3 through 5, and overall) and Common Toxicity Criteria grade (any grade and grade 3 to 5 , on a scale of 1 to 5 , with higher numbers indicating worse toxic effects). There was a higher incidence of thromboembolic events among women who were assigned to one of the regimens that included tamoxifen than among those who were assigned to letrozole monotherapy (4.1 to $4.9 \%$ vs. $2.4 \%, \mathrm{P}<0.001)$. There were similar rates of stroke and transient cerebral ischemic attack between women who were assigned to one of the regimens that included tamoxifen and those who were not (1.7 to $1.9 \%$ and $1.4 \%$, respectively; $\mathrm{P}=0.74)$. The incidence of cardiac events of any type or grade was similar between women who were assigned to one of the regimens that included letrozole and women who were assigned to tamoxifen monotherapy (6.1 to $7.0 \%$ and $5.7 \%$, respectively; $\mathrm{P}=0.45$ ). The incidence of hypercholesterolemia (predominantly mild) was lower among women who were assigned to tamoxifen monotherapy than among those who were assigned to one of the regimens that included letrozole ( $29.9 \%$ vs. 41.4 to $53.2 \%, \mathrm{P}<0.001$ ).

Vaginal bleeding was reported in $9.9 \%$ of the women who were assigned to tamoxifen monotherapy, 5.1\% of those who were assigned to letrozole monotherapy, and 6.4 to $7.5 \%$ of those who were assigned to sequential therapy $(\mathrm{P}<0.001)$. Hot flashes and night sweats occurred in all groups but were more frequent among women who were assigned to one of the regimens that included tamoxifen than among women assigned to letrozole monotherapy (hot flashes: 41.7 to $44.0 \%$ of women vs. $37.7 \%, P=0.003$; night sweats: 17.8 to $19.4 \%$ vs. $15.6 \%, P=0.04)$. Arthralgia, myalgia, or both were more frequent among women assigned to one of the regimens that included letrozole than among women assigned to tamoxifen monotherapy (31.9 to $34.7 \%$ of women vs. $30.1 \%, P=0.05$ ), and the excess incidence among women in the 


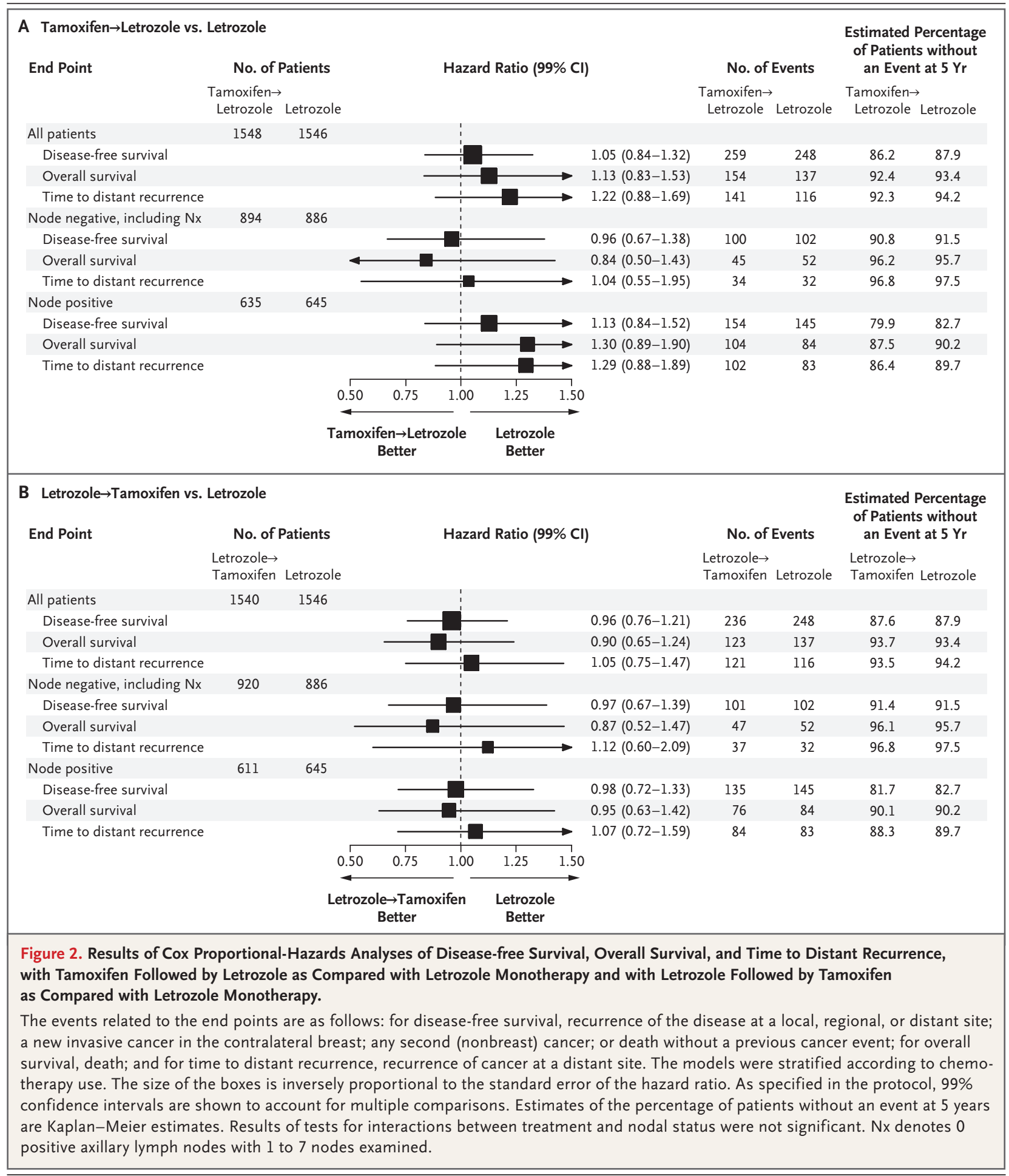

sequential regimens was seen during the periods women assigned to letrozole monotherapy and when the women were receiving letrozole (Sec- lowest among women assigned to tamoxifen tion 4 in the Supplementary Appendix).

The incidence of fractures was highest among among women assigned to tamoxifen followed by 


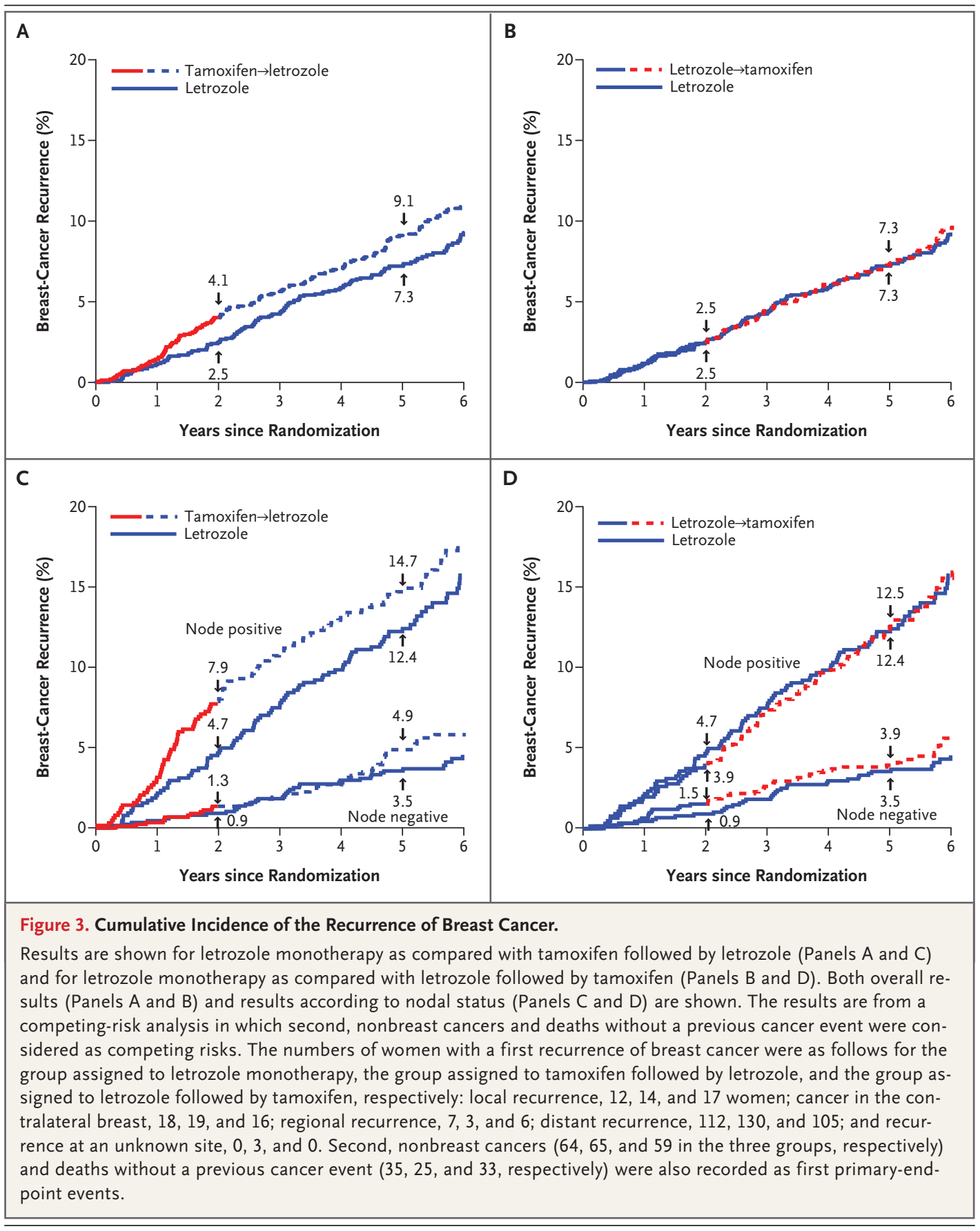

letrozole was similar to that among women assigned to letrozole alone $(9.4 \%$ and $9.8 \%$, respectively), and the incidence of fractures in the group assigned to letrozole followed by tamoxifen was similar to that among women assigned to tamoxifen alone (7.5\% and $7.3 \%$, respectively). Among the four groups, there was a similar number of deaths without a recurrence of breast cancer and of second (nonbreast) primary cancers, except for endometrial cancers, of which there were 13 cases in the group assigned to tamoxifen monotherapy, 2 cases in the group assigned to letrozole monotherapy, and 4 cases in each sequential group $(\mathrm{P}=0.01)$. 
UPDATED ANALYSIS OF MONOTHERAPY

The median follow-up period for the analysis that included women assigned to letrozole or tamoxifen monotherapy in both randomization options was 76 months. In the updated intention-to-treat analyses comparing letrozole monotherapy with tamoxifen monotherapy, there were 509 primaryend-point events in the letrozole group versus 565 events in the tamoxifen group $(\mathrm{P}=0.03)$. The time to distant recurrence also differed significantly in favor of letrozole ( $\mathrm{P}=0.05)$ (Fig. 4). The 5-year overall survival was $91.8 \%$ in the letrozole group and $90.9 \%$ in the tamoxifen group (hazard ratio, 0.87; $95 \%$ confidence interval, 0.75 to $1.02 ; \mathrm{P}=0.08$ ) (Fig. 4). The censored analyses (Fig. 4) suggested that there was more benefit with letrozole for each end point, but these analyses were subject to biases, some of which may have favored letrozole. The difference in the incidence of adverse events between the monotherapy groups changed little from that previously reported ${ }^{3}$ (data not shown). Eighty-seven deaths without prior cancer events were recorded in each monotherapy group. Section 5 in the Supplementary Appendix gives further details on the types of first primary-end-point events and the selective crossover.

\section{DISCUSSION}

The main purpose of the BIG 1-98 trial was to compare an aromatase inhibitor (letrozole) with tamoxifen as adjuvant therapy in postmenopausal women with endocrine-responsive early breast cancer. We report here analyses of letrozole monotherapy as compared with sequential treatment with tamoxifen and letrozole; in addition, we investigated whether letrozole monotherapy prolongs overall survival as compared with tamoxifen monotherapy. The limitations of the study include the selective crossover to letrozole among women assigned to tamoxifen monotherapy and the inability, after a median follow-up period of 6 years, to assess the influence of a potential carryover effect of letrozole on the results.

In the analyses of sequential treatments, neither tamoxifen followed by letrozole nor letrozole followed by tamoxifen showed superiority over letrozole alone. A previous analysis of the trial data $^{23}$ showed that the frequency of relapses within 2 years after randomization was significantly reduced with letrozole as compared with tamoxifen, especially among women with many involved lymph nodes, large tumors, or vascular invasion. A similar pattern was seen in the current analysis of the sequential-treatment cohort. There was a nonsignificant increase in the risk of early relapse among women with node-positive disease who were assigned to tamoxifen followed by letrozole (Fig. 3C). Since the interactions between treatment group and nodal status were not significant, caution is required in the interpretation of these subgroup analyses. ${ }^{24}$

The present analysis shows that treatment with letrozole for 2 years followed by tamoxifen yielded outcomes similar to those seen with letrozole monotherapy. It is possible that part of this effect is a carryover benefit of the initial letrozole therapy, similar to that observed after cessation of anastrozole in the ATAC study. ${ }^{4}$ The follow-up of the BIG 1-98 trial is ongoing; the follow-up data that are currently available (median, 6 years) indicate that, after 2 years of adjuvant therapy with letrozole, a switch to tamoxifen to complete 5 years of therapy would be acceptable, if cessation of letrozole is required for any reason.

Previous analyses of the BIG 1-98 trial have shown that, as compared with tamoxifen alone, letrozole monotherapy significantly reduces the risk of recurrence of disease, especially at distant sites. $^{2,3}$ The updated intention-to-treat analysis of monotherapy confirms these observations and shows a nonsignificant difference between letrozole monotherapy and tamoxifen monotherapy with respect to overall survival ( $\mathrm{P}=0.08)$. Our belief that this result underestimates the survival benefit that would have accrued if there had been no crossover to letrozole is based on evidence from independent trials that have shown a survival benefit from switching to an aromatase inhibitor after initial treatment with tamoxifen. ${ }^{11,13}$ The censored analysis of overall survival, which suggests an even greater advantage of letrozole over tamoxifen than that seen in the intention-to-treat analysis (Fig. 4), may be an overestimate, particularly since women who had recurrent disease were not candidates for crossover (see Section 5 in the Supplementary Appendix). Thus, it is likely that the best estimate of the survival benefit with letrozole if there had been no selective crossover lies somewhere between these two extremes.

After 5 years of adjuvant endocrine therapy, relapses continue to occur in women with endocrineresponsive early breast cancer. Other trials have shown the value of extended therapy with an aro- 


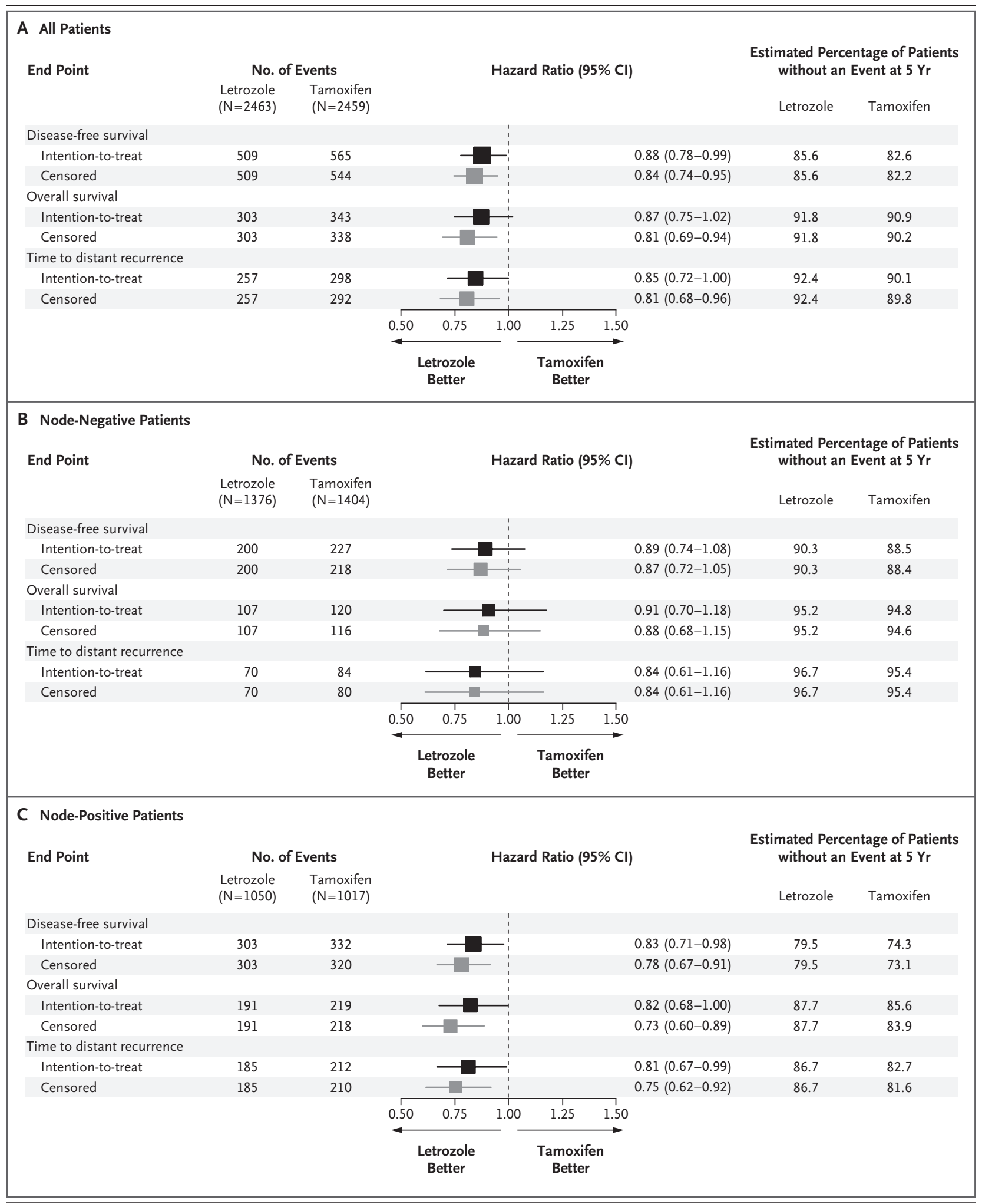


Figure 4 (facing page). Results of Cox ProportionalHazards Analyses of Disease-free Survival, Overall Survival, and Time to Distant Recurrence, with Letrozole Monotherapy as Compared with Tamoxifen Monotherapy among Women Assigned to the Single-Agent Treatment Groups.

Results are shown for the total cohort and according to nodal status. Results of the intention-to-treat and censored analyses are presented. The events related to the end points are as follows: for disease-free survival, recurrence of the disease at a local, regional, or distant site; a new invasive cancer in the contralateral breast; any second (nonbreast) cancer; or death without a previous cancer event; for overall survival, death; and for time to distant recurrence, recurrence of cancer at a distant site. The models were stratified according to study cohort (two-group or four-group randomization option) and chemotherapy use. The size of the boxes is inversely proportional to the standard error of the hazard ratio. Estimates of the percentage of patients without an event at 5 years are Kaplan-Meier estimates. The results of tests for interactions between treatment and nodal status were not significant. The intention-totreat analysis included all women and all follow-up time and events according to treatment assignment. The exploratory censored analysis was identical except that it excluded (i.e., censored) events and follow-up beyond the time of selective crossover among women randomly assigned to tamoxifen. Both analyses were subject to potential biases that may have influenced the estimated magnitude of the benefit with letrozole as compared with tamoxifen.

matase inhibitor after 5 years of adjuvant therapy with tamoxifen. ${ }^{25,26}$ The ongoing Study of Letrozole Extension (SOLE; NCT00553410) ${ }^{27}$ is exploring this concept in more detail and is adding an evaluation of intermittent letrozole as extended adjuvant therapy. ${ }^{28,29}$

The adverse-event profile and the number of deaths without a previous cancer event in the monotherapy and the sequential-therapy groups are reassuring. There were no unexpected lifethreatening adverse events in any group. The safety and efficacy results add to the information that supports the use of adjuvant endocrine therapy with letrozole in postmenopausal women with endocrine-responsive early breast cancer and provide additional treatment options for such women.

Supported by Novartis. The International Breast Cancer Study Group (IBCSG), which coordinated the study, is also supported by the Swedish Cancer Society, the Cancer Council Australia, the Australian New Zealand Breast Cancer Trials Group, the Frontier Science and Technology Research Foundation, the Swiss Group for Clinical Cancer Research, the National Cancer Institute (CA75362), Cancer Research Switzerland and Oncosuisse, and the Foundation for Clinical Cancer Research of Eastern Switzerland.

Dr. Mouridsen reports receiving consulting and lecture fees from Novartis; Dr. Thürlimann, owning stock in Novartis; Dr. Mauriac, receiving consulting fees from Novartis and AstraZeneca and grant support from AstraZeneca; Dr. Forbes, receiving consulting fees from Eli Lilly, Novartis, and AstraZeneca and lecture fees from AstraZeneca; Dr. Smith, receiving consulting and lecture fees from Novartis; and Dr. Goldhirsch, receiving consulting and lecture fees from Novartis, AstraZeneca, and Pfizer. Novartis contracted with the IBCSG for provision of services related to the conduct and management of the trial. Drs. Goldhirsch, Coates, and Gelber are responsible for the scientific management of the IBCSG. No other potential conflict of interest relevant to this article was reported.

We thank the women, physicians, nurses, and data managers who participated in this clinical trial; the secretariat of the Breast International Group (BIG); the BIG groups, including the IBCSG, with participating centers from Australia and New Zealand (members of the Australian New Zealand Breast Cancer Trials Group), Brazil, Chile (members of the Chilean Cooperative Group for Oncologic Research), Hungary, Italy, Peru, Slovenia, South Africa, Sweden (members of the West Swedish Breast Cancer Study Group), Switzerland (members of the Swiss Group for Clinical Cancer Research), and United Kingdom; the Danish Breast Cancer Cooperative Group; the French Group (Federation Nationale des Centres de Lutte contre le Cancer); and the North Yorkshire Group; and independent centers and groups from Argentina, Australia, Belgium, Canada, Chile, Czech Republic, Germany, Hungary, Italy, the Netherlands, New Zealand, Poland, Portugal, Russia, South Africa, Spain, Switzerland, Turkey, United Kingdom, and Uruguay.

\section{APPENDIX}

The affiliations of the Writing Committee are as follows: Danish Breast Cancer Group, Rigshospitalet, Vejle Hospital, Copenhagen (H.M.); the International Breast Cancer Study Group (IBCSG) Statistical Center, Dana-Farber Cancer Institute (A.G.-H., K.N.P., M.M.R., R.D.G.), Harvard School of Public Health and Harvard Medical School (M.M.R., R.D.G.), and Frontier Science and Technology Research Foundation (K.N.P., R.D.G.) — all in Boston; the European Institute of Oncology, Milan (A.G.); the Oncology Institute of Southern Switzerland, Bellinzona (A.G.), the Breast Center, Kantonsspital, St. Gallen (B.T.), and the Swiss Group for Clinical Cancer Research, Bern (B.T.) — all in Switzerland; the Department of Medical Oncology, University Hospital Gasthuisberg, Catholic University of Leuven, Leuven, Belgium (R.P.); the Royal Marsden Hospital, London, and the Royal Marsden National Health Service Trust, Sutton, Surrey both in the United Kingdom (I.S.); Fédération Nationale des Centres de Lutte contre le Cancer, Institut Bergonié, Bordeaux, France (L.M.); and the Australian New Zealand Breast Cancer Trials Group, University of Newcastle, Calvary Mater Newcastle, Newcastle, NSW (J.F.F.), and the University of Sydney, Sydney (A.S.C.) — both in Australia. 
REFERENCES

1. Early Breast Cancer Trialists' Collaborative Group. Effects of chemotherapy and hormonal therapy for early breast cancer on recurrence and 15-year survival: an overview of the randomised trials. Lancet 2005;365:1687-717.

2. The Breast International Group (BIG) 1-98 Collaborative Group. A comparison of letrozole and tamoxifen in postmenopausal women with early breast cancer. N Engl J Med 2005;353:2747-57.

3. Coates AS, Keshaviah A, Thürlimann $\mathrm{B}$, et al. Five years of letrozole compared with tamoxifen as initial adjuvant therapy for postmenopausal women with endocrine-responsive early breast cancer: update of study BIG 1-98. J Clin Oncol 2007; 25:486-92.

4. Forbes JF, Cuzick J, Buzdar A, Howell A, Tobias JS, Baum M. Effect of anastrozole and tamoxifen as adjuvant treatment for early-stage breast cancer: 100-month analysis of the ATAC trial. Lancet Oncol 2008;9:45-53

5. Baum M, Budzar AU, Cuzick J, et al. Anastrozole alone or in combination with tamoxifen versus tamoxifen alone for adjuvant treatment of postmenopausal women with early breast cancer: first results of the ATAC randomised trial. Lancet 2002; 359:2131-9. [Erratum, Lancet 2002;360: 1520.]

6. Coombes RC, Hall E, Gibson LJ, et al. A randomized trial of exemestane after two to three years of tamoxifen therapy in postmenopausal women with primary breast cancer. N Engl J Med 2004;350:108192. [Erratum, N Engl J Med 2004;351: 2461.]

7. Jakesz R, Jonat $\mathrm{W}$, Gnant $\mathrm{M}$, et al. Switching of postmenopausal women with endocrine-responsive early breast cancer to anastrozole after 2 years' adjuvant tamoxifen: combined results of ABCSG trial 8 and ARNO 95 trial. Lancet 2005;366: 455-62.

8. Kaufmann M, Jonat W, Hilfrich J, et al. Improved overall survival in postmenopausal women with early breast cancer after anastrozole initiated after treatment with tamoxifen compared with continued tamoxifen: the ARNO 95 Study. J Clin Oncol 2007;25:2664-70.

9. Boccardo F, Rubagotti A, Puntoni M, et al. Switching to anastrozole versus continued tamoxifen treatment of early breast cancer: preliminary results of the Italian
Tamoxifen Anastrozole Trial. J Clin Oncol 2005;23:5138-47.

10. Boccardo F. Switching to anastrozole after tamoxifen improves survival in postmenopausal women with breast cancer. Nat Clin Pract Oncol 2008;5:76-7.

11. Coombes RC, Kilburn LS, Snowdon $\mathrm{CF}$, et al. Survival and safety of exemestane versus tamoxifen after 2-3 years' tamoxifen treatment (Intergroup Exemestane Study): a randomised controlled trial. Lancet 2007;369:559-70. [Erratum, Lancet 2007; 369:906.]

12. Jonat W, Gnant M, Boccardo F, et al. Effectiveness of switching from adjuvan tamoxifen to anastrozole in postmenopausal women with hormone-sensitive earlystage breast cancer: a meta-analysis. Lancet Oncol 2006;7:991-6. [Erratum, Lancet Oncol 2007;8:6.]

13. Ingle JN, Dowsett M, Cuzick J, Davies C. Aromatase inhibitors versus tamoxifen as adjuvant therapy for postmenopausal women with estrogen receptor positive breast cancer: meta-analyses of randomized trials of monotherapy and switching strategies. Cancer Res 2009;69:Suppl:12. abstract.

14. Winer EP, Hudis C, Burstein HJ, et al American Society of Clinical Oncology technology assessment on the use of aromatase inhibitors as adjuvant therapy for postmenopausal women with hormone receptor-positive breast cancer: status report 2004. J Clin Oncol 2005;23:619-29.

15. Goldhirsch A, Wood WC, Gelber RD, et al. Progress and promise: highlights of the international expert consensus on the primary therapy of early breast cancer 2007. Ann Oncol 2007;18:1133-44. [Erratum, Ann Oncol 2007;18:1917.

16. Carlson RW, Brown E, Burstein HJ, et al. NCCN Task Force report: adjuvant therapy for breast cancer. J Natl Compr Canc Netw 2006;4:Suppl 1:S1-S26

17. Cuzick J, Sasieni P, Howell A. Should aromatase inhibitors be used as initial adjuvant treatment or sequenced after tamoxifen? Br J Cancer 2006;94:460-4

18. Punglia RS, Kuntz KM, Winer EP, Weeks JC, Burstein HJ. Optimizing adjuvant endocrine therapy in postmenopausal women with early-stage breast cancer a decision analysis. J Clin Oncol 2005, 23:5178-87.

19. Giobbie-Hurder A, Price KN, Gelber $\mathrm{RD}$. Design, conduct, and analyses of Breas
International Group (BIG) 1-98: a randomized, double-blind, phase-III study comparing letrozole and tamoxifen as adjuvant endocrine therapy for postmenopausal women with receptor-positive, early breast cancer. Clin Trials 2009;6:272-87.

20. Kaplan EL, Meier P. Nonparametric estimation from incomplete observations. J Am Stat Assoc 1958;53:457-81.

21. Cox DR. Regression models and lifetables. J R Stat Soc [B] 1972;34:187-220.

22. Gray RJ. A class of k-sample tests for comparing the cumulative incidence of a competing risk. Ann Stat 1988;16:114154.

23. Mauriac L, Keshaviah A, Debled M, et al. Predictors of early relapse in postmenopausal women with hormone receptor-positive breast cancer in the BIG 1-98 trial. Ann Oncol 2007;18:859-67.

24. Wang R, Lagakos SW, Ware JH, Hunter DJ, Drazen JM. Statistics in medicine - reporting of subgroup analyses in clinical trials. N Engl J Med 2007;357:218994.

25. Goss PE, Ingle JN, Martino S, et al. A randomized trial of letrozole in postmenopausal women after five years of tamoxifen therapy for early-stage breast cancer. N Engl J Med 2003;349:1793-802.

26. Mamounas EP, Jeong JH, Wickerham DL, et al. Benefit from exemestane as extended adjuvant therapy after 5 years of adjuvant tamoxifen: intention-to-treat analysis of the National Surgical Adjuvant Breast and Bowel Project B-33 trial. J Clin Oncol 2008;26:1965-71.

27. Clinical Trials (PDQ). Phase III randomized study of continuous letrozole versus intermittent letrozole in postmenopausal women with hormone receptorpositive, node-positive, early-stage breast cancer after completion of 4 to 6 years of prior adjuvant endocrine therapy. Bethesda, MD: National Cancer Institute. (Accessed July 24, 2009, at http://www.cancer.gov/ clinicaltrials/IBCSG-35-07.)

28. Jordan VC. The 38th David A. Karnofsky lecture: the paradoxical actions of estrogen in breast cancer - survival or death? J Clin Oncol 2008;26:3073-82.

29. Sabnis GJ, Macedo LF, Goloubeva O, Schayowitz A, Brodie AM. Stopping treatment can reverse acquired resistance to letrozole. Cancer Res 2008;68:4518-24. Copyright (c) 2009 Massachusetts Medical Society. 